Y. HIRASAWA

KODAI MATH. J.

7 (1984), 34-55

\title{
ON THE CONSTRUCTION OF LINEARLY INDEPENDENT VECTORS WITH VARIABLE COMPONENTS
}

\author{
By YoshiKazU HIRASAwA
}

\section{$\S 1$. Introduction.}

We use the same notations as in a previous paper [1]. Let $J$ be a closed interval $[\gamma, \delta]=\{t \mid \gamma \leqq t \leqq \delta, t \in \mathbf{R}\}$. Let $C^{\mu}(J, \mathbf{C})$ denote the totality of complexvalued functions defined and of class $\mathrm{C}^{\mu}$ on $J(\mu=0,1, \cdots, \infty)$. Hereafter we fix some $\mu$.

For the sake of brevity, we denote $C^{\mu}(J, \mathbf{C})$ by $K(J)$, and $K(J)^{n}$ by $M(J)$ :

$$
M(J)=\left\{\boldsymbol{f}(t)=\operatorname{col}\left(f_{1}(t), f_{2}(t), \cdots, f_{n}(t)\right) \mid f_{j}(t) \in K(J), j=1,2, \cdots, n\right\} .
$$

Let $X(t)$ be an $n \times h$ matrix whose components all belong to $K(J)$ :

$$
X(t)=\left(\begin{array}{cccc}
x_{11}(t) & x_{12}(t) & \cdots & x_{1 h}(t) \\
x_{21}(t) & x_{22}(t) & \cdots & x_{2 h}(t) \\
\vdots & \vdots & & \vdots \\
x_{n 1}(t) & x_{n 2}(t) & \cdots & x_{n h}(t)
\end{array}\right),
$$

where $h$ is an integer such that $1 \leqq h \leqq n-1$, and suppose that a condition

$$
\operatorname{rank} X(t)=h
$$

is satisfied on $J$.

The first purpose of this paper is to prove the following theorem:

THEOREM 1. Let $X(t)$ be the $n \times h$ matrix given above and satisfying the condition (1.2) on $J$. Then there exists a vector $\boldsymbol{y}(t) \in M(J)$ such that

$$
\left\{\begin{array}{l}
\operatorname{rank} \boldsymbol{y}(t)=1 \quad \text { on } J, \\
\operatorname{rank}(X(t), \boldsymbol{y}(t))=h+1 \quad \text { on } J .
\end{array}\right.
$$

As a corollary of Theorem 1, we obtain immediately the following theorem:

THEOREM 2. Let $X(t)$ be the $n \times h$ matrix given above and satisfying the condition (1.2) on $J$. Then there exists an $n \times(n-h)$ matrix $Y(t)$ whose components

Received March 1, 1983 
ail belong to $K(J)$ :

$$
Y(t)=\left(\begin{array}{cccc}
y_{1, h+1}(t) & y_{1, h+2}(t) & \cdots & y_{1 n}(t) \\
y_{2, h+1}(t) & y_{2, h+2}(t) & \cdots & y_{2 n}(t) \\
\vdots & \vdots & & \vdots \\
y_{n, h+1}(t) & y_{n, h+2}(t) & \cdots & y_{n n}(t)
\end{array}\right)
$$

such that

$$
\begin{cases}\operatorname{rank} Y(t)=n-h & \text { on } J, \\ \operatorname{rank}(X(t), Y(t))=n & \text { on } J .\end{cases}
$$

Now, let $I$ be a closed interval $[\alpha, \beta]=\{t \mid \alpha \leqq t \leqq \beta, t \leqq \mathbf{R}\}$ and let $B(t)$ be a square matrix of degree $n$ whose components all belong to $K(I)$ :

$$
B(t)=\left(\begin{array}{cccc}
b_{11}(t) & b_{12}(t) & \cdots & b_{1 n}(t) \\
b_{21}(t) & b_{22}(t) & \cdots & b_{2 n}(t) \\
\vdots & \vdots & & \vdots \\
b_{n 1}(t) & b_{n 2}(t) & \cdots & b_{n n}(t)
\end{array}\right) .
$$

We assume that for a positive integer $s: 2 \leqq s \leqq n-1$, a condition

$$
\operatorname{rank} B(t)=n-s(=r)
$$

is satisfied on $I$, and consider a linear equation

$$
B(t) \boldsymbol{f}(t)=\boldsymbol{o} \quad \text { on } I ; \quad \boldsymbol{f}(t) \in M(I) .
$$

We denote the totality of solutions of (1.8) by $W(I)$ :

$$
W(I)=\{\boldsymbol{f}(t) \in M(I) \mid B(t) \boldsymbol{f}(t)=\boldsymbol{o} \text { on } I\} .
$$

Then, we know that there exist $s$ vectors $\boldsymbol{x}_{1}(t), \boldsymbol{x}_{2}(t), \cdots, \boldsymbol{x}_{s}(t)$ belonging to $W(I)$, such that

$$
\operatorname{rank}\left(\boldsymbol{x}_{1}(t), \boldsymbol{x}_{2}(t), \cdots, \boldsymbol{x}_{s}(t)\right)=s \text { on } I \text {. }
$$

For the proof of this fact, see, for example, the proof of Theorem in the previous paper [1].

The second purpose of this paper is to prove the following theorem:

THEOREM 3. Let $\boldsymbol{x}_{1}(t), \boldsymbol{x}_{2}(t), \cdots, \boldsymbol{x}_{s^{\prime}}(t)$ be $s^{\prime}$ prescribed vectors belonging to $W(I)$ and satisfying a condition

$$
\operatorname{rank}\left(\boldsymbol{x}_{1}(t), \boldsymbol{x}_{2}(t), \cdots, \boldsymbol{x}_{s^{\prime}}(t)\right)=s^{\prime} \text { on } I,
$$

where $s^{\prime}$ is a positive integer such that $1 \leqq s^{\prime}<s$.

Then there exist $\left(s-s^{\prime}\right)$ vectors $\boldsymbol{y}_{s^{\prime}+1}(t), \boldsymbol{y}_{s^{\prime}+2}(t), \cdots, \boldsymbol{y}_{s}(t)$ belonging to $W(I)$ and satisfying conditions 


$$
\left\{\begin{array}{l}
\operatorname{rank}\left(\boldsymbol{y}_{s^{\prime}+1}(t), \cdots, \boldsymbol{y}_{s}(t)\right)=s-s^{\prime} \quad \text { on } I, \\
\operatorname{rank}\left(\boldsymbol{x}_{1}(t), \cdots, \boldsymbol{x}_{s^{\prime}}(t), \boldsymbol{y}_{s^{\prime}+1}(t), \cdots, \boldsymbol{y}_{s}(t)\right)=s \text { on } I .
\end{array}\right.
$$

In general, we denote a minor of degree $r$ of the matrix $B(t)$ which is given by (1.6), by

$$
\begin{aligned}
& B\left(\begin{array}{cccc}
j_{1} & j_{2} & \cdots & j_{r} \\
k_{1} & k_{2} & \cdots & k_{r}
\end{array}\right)=\left|\begin{array}{cccc}
b_{j_{1} k_{1}}(t) & b_{j_{1} k_{2}}(t) & \cdots & b_{j_{1} k_{r}}(t) \\
b_{j_{2} k_{1}}(t) & b_{j_{2} k_{2}}(t) & \cdots & b_{j_{2} k_{r}}(t) \\
\vdots & \vdots & & \vdots \\
b_{j_{r} k_{1}}(t) & b_{j_{r} k_{2}}(t) & \cdots & b_{\jmath_{r} k_{r}}(t)
\end{array}\right| \\
& \left(\begin{array}{l}
1 \leqq j_{1}<j_{2}<\cdots<j_{r} \leqq n \\
1 \leqq k_{1}<k_{2}<\cdots<k_{r} \leqq n
\end{array}\right),
\end{aligned}
$$

and then, a minor of degree $h$ of the $n \times h$ matrix $X(t)$ which is given by (1.1). is especially denoted by

$$
\begin{gathered}
X\left(\begin{array}{cccc}
k_{1} & k_{2} & \cdots & k_{h} \\
1 & 2 & \cdots & h
\end{array}\right)=\left|\begin{array}{cccc}
x_{k_{11}}(t) & x_{k_{12}}(t) & \cdots & x_{k_{1} h}(t) \\
x_{k_{21}(t)} & x_{k_{2} 2}(t) & \cdots & x_{k_{2} h}(t) \\
\vdots & \vdots & & \vdots \\
x_{k_{h^{1}}}(t) & x_{k_{h^{2}}}(t) & \cdots & x_{k_{h} h}(t)
\end{array}\right| \\
\left(1 \leqq k_{1}<k_{2}<\cdots<k_{h} \leqq n\right) .
\end{gathered}
$$

In $\S 2$, we shall give two lemmas which will be used for the proof of Theorem 1 , and in $\S 3$, we shall prove Theorem 1 .

In $\S 4$, we shall give a summary of the matters which are necessary for the proof of Theorem 3, and in $\S \S 5-6$, we shall prove Theorem 3 .

\section{$\S 2$. Lemmas.}

LEMMA 1. Let $J_{0}$ be a closed interval $\left[Y_{0}, \delta_{0}\right]=\left\{t \mid \gamma_{0} \leqq t \leqq \delta_{0}, t \in \mathbf{R}\right\}$ and let $\varphi_{\tau}(t)\left(\tau=1,2, \cdots, \tau_{0}\right)$ be a finite number of real-valued continuous functions defined on $J_{0}$. Then there exists a closed interval $J^{*}=\left[\gamma^{*}, \delta^{*}\right]$ contained in $J_{0}$, such that each of $\varphi_{\tau}(t)\left(\tau=1,2, \cdots, \tau_{0}\right)$ is one-signed or identically equal to zero on $J^{*}$ respectively.

Proof. Put

$$
\begin{aligned}
& E_{+}^{(1)}=\left\{t \in J_{0} \mid \varphi_{1}(t)>0\right\}, \quad E_{-}^{(1)}=\left\{t \in J_{0} \mid \varphi_{1}(t)<0\right\}, \\
& E_{0}^{(1)}=\left\{t \in J_{0} \mid \varphi_{1}(t)=0\right\} .
\end{aligned}
$$

Then, $E_{+}^{(1)}, E_{-}^{(1)}$ and $E_{0}^{(1)}$ are disjoint with each other and $E_{+}^{(1)} \cup E_{-}^{(1)} \cup E_{0}^{(1)}$ $=J_{0} . \quad E_{+}^{(1)}$ and $E_{-}^{(1)}$ are relatively open on $J_{0}$. Therefore, if $E_{+}^{(1)} \neq \varnothing$ or $E_{-}^{(1)} \neq \varnothing$, we can find a closed interval $J_{1}^{*}=\left[\gamma_{1}^{*}, \delta_{1}^{*}\right] \subset J_{0}$ such that $\varphi_{1}(t)>0$ or $\varphi_{1}(t)<0$ on $J_{1}^{*}$. If $E_{+}^{(1)}=\varnothing$ and $E_{-}^{(1)}=\varnothing$, we see $\phi_{1}(t) \equiv 0$ on $J_{1}^{*}=J_{0}$.

By repeating the process just described, for the interval $J_{1}^{*}$ and the functions 
$\varphi_{\tau}(t)\left(\tau=2,3, \cdots, \tau_{0}\right)$ successively, we obtain the desired interval $J^{*}=\left[\gamma^{*}, \delta^{*}\right]$.

Now, for any value $t_{0} \in \mathbf{R}$, we put

$$
\begin{aligned}
& e_{+}\left(t ; t_{0}\right)=\left\{\begin{array}{cc}
0 & \text { for } t \leqq t_{0}, \\
\exp \left\{-\frac{1}{\left(t-t_{0}\right)^{2}}\right\} & \text { for } t>t_{0}
\end{array}\right. \\
& e_{-}\left(t ; t_{0}\right)=\left\{\begin{array}{cc}
\exp \left\{-\frac{1}{\left(t-t_{0}\right)^{2}}\right\} & \text { for } t<t_{0}, \\
0 & \text { for } t \geqq t_{0},
\end{array}\right.
\end{aligned}
$$

and for any values $t_{1}, t_{2} \in \mathbf{R}$ such that $t_{1}<t_{2}$, we put

$$
e\left(t ; t_{1}, t_{2}\right)= \begin{cases}0 & \text { for } t \leqq t_{1}, \\ \exp \left\{-\frac{1}{\left(t-t_{1}\right)^{2}}-\frac{1}{\left(t-t_{2}\right)^{2}}\right\} & \text { for } t_{1}<t<t_{2}, \\ 0 & \text { for } t \geqq t_{2} .\end{cases}
$$

Then we see that the functions $e_{+}\left(t ; t_{0}\right), e_{-}\left(t ; t_{0}\right)$ and $e\left(t ; t_{1}, t_{2}\right)$ belong to $C^{\infty}(\mathbf{R}, \mathbf{R})$.

Next, let $J_{1}=\left(\gamma_{1}, \delta_{1}\right)$ and $J_{2}=\left(\gamma_{2}, \delta_{2}\right)$ be open intervals on $\mathbf{R}$ such that $\gamma_{1}<\gamma_{2}<\delta_{1}<\delta_{2}$

Furthermore let $\theta(t)$ and $\omega(t)$ be functions belonging to $K\left(\bar{J}_{1}\right)$ and to $K\left(\bar{J}_{2}\right)$ respectively, such that each of $\theta_{(r)}(t)(=\operatorname{Re} \theta(t)), \quad \theta_{(i)}(t)(=\operatorname{Im} \theta(t)), \quad \omega_{(r)}(t)$ $(=\operatorname{Re} \omega(t))$ and $\omega_{(i)}(t)(=\operatorname{Im} \omega(t))$ is one-signed or identically equal to zero on $\bar{J}_{1} \cap \bar{J}_{2}=\left[\gamma_{2}, \delta_{1}\right]$.

Under these circumstances, we shall prove the following lemma:

LEMma 2. Let

$$
f(t)=\left(c_{1}+i d_{1}\right) e\left(t ; \gamma_{1}, \delta_{1}\right) ; i=\sqrt{-1},
$$

where $c_{1}$ and $d_{1}$ are real non-zero constants. Then, there exist two real non-zero constants $c_{2}$ and $d_{2}$ such that a function

satisfies conditions

$$
g(t)=\left(c_{2}+i d_{2}\right) e\left(t ; \gamma_{2}, \delta_{2}\right)
$$

$$
g(t)-\omega(t) f(t) \neq 0 \quad \text { on } J_{2}=\left(\gamma_{2}, \delta_{2}\right)
$$

and

$$
f(t)-\theta(t) g(t) \neq 0 \quad \text { on } J_{1}=\left(\gamma_{1}, \delta_{1}\right)
$$

Proof. We, at the beginning, take note of the fact that the functions $\theta_{(r)}(t), \theta_{(i)}(t), \omega_{(r)}(t)$ and $\omega_{(i)}(t)$ are continuous and bounded on the interval 
$\bar{J}_{1} \cap \bar{J}_{2}=\left[\gamma_{2}, \delta_{1}\right]$.

Let us put

$$
f_{(r)}(t)=\operatorname{Re} f(t), \quad f_{(\imath)}(t)=\operatorname{Im} f(t), \quad g_{(r)}(t)=\operatorname{Re} g(t), \quad g_{(i)}(t)=\operatorname{Im} g(t) .
$$

We show first that by choosing either $c_{2}$ or $d_{2}$ suitably, we can make the function $g(t)$ satisfy the condition (2.1).

Since $\omega(t) f(t) \equiv 0$ on the interval $\left[\delta_{1}, \delta_{2}\right)$, we have only to determine the nonzero constants $c_{2}$ and $d_{2}$, such that the condition (2.1) is satisfied on the interval $J_{1} \cap J_{2}=\left(\gamma_{2}, \delta_{1}\right)$ instead of $J_{2}$.

For the determination of the constants $c_{2}$ and $d_{2}$, we shall distinguish four cases, according to the values of $\omega_{(r)}(t)$ and $\omega_{(i)}(t)$ on $\bar{J}_{1} \cap \bar{J}_{2}$ :

Case I-( i ) $\omega(t) \equiv 0 \quad$ on $\bar{J}_{1} \cap \bar{J}_{2}$,

Case I-(ii) $\omega_{(r)}(t) \neq 0$ and $\omega_{(i)}(t) \equiv 0$ on $\bar{J}_{1} \cap \bar{J}_{2}$,

Case I-(iii) $\omega_{(r)}(t) \equiv 0$ and $\omega_{(i)}(t) \neq 0$ on $\bar{J}_{1} \cap \bar{J}_{2}$,

Case I-(iv) $\omega_{(r)}(t) \neq 0$ and $\omega_{(i)}(t) \neq 0$ on $\bar{J}_{1} \cap \bar{J}_{2}$.

In Case I-(i), the condition (2.1) is satisfied for all non-zero values of $c_{2}$ and $d_{2}$, because we have $\omega(t) f(t) \equiv 0$ on $\bar{J}_{2}$.

In Case I-(ii), since

$$
\begin{array}{ll}
\operatorname{Re} \omega(t) f(t)=\omega_{(r)}(t) f_{(r)}(t)=c_{1} \omega_{(r)}(t) e\left(t ; \gamma_{1}, \delta_{1}\right) & \text { on } \bar{J}_{1} \cap \bar{J}_{2} ; \\
\operatorname{Im} \omega(t) f(t)=\omega_{(r)}(t) f_{(i)}(t)=d_{1} \omega_{(r)}(t) e\left(t ; \gamma_{1}, \delta_{1}\right) & \text { on } \bar{J}_{1} \cap \bar{J}_{2},
\end{array}
$$

the condition (2.1) is satisfied, if we choose either the constant $c_{2}$ with the opposite $\operatorname{sign}$ to $c_{1} \omega_{(r)}(t)$ on $\bar{J}_{1} \cap \bar{J}_{2}$, or the constant $d_{2}$ with the opposite sign to $d_{1} \omega_{(r)}(t)$ on $\bar{J}_{1} \cap \bar{J}_{2}$.

In Case I-(iii), since

$$
\begin{array}{ll}
\operatorname{Re} \omega(t) f(t)=-\omega_{(i)}(t) f_{(i)}(t)=-d_{1} \omega_{(2)}(t) e\left(t ; \gamma_{1}, \delta_{1}\right) & \text { on } \bar{J}_{1} \cap \bar{J}_{2} ; \\
\operatorname{Im} \omega(t) f(t)=\omega_{(\imath)}(t) f_{(r)}(t)=c_{1} \omega_{(i)}(t) e\left(t ; \gamma_{1}, \delta_{1}\right) & \text { on } \bar{J}_{1} \cap \bar{J}_{2},
\end{array}
$$

the condition (2.1) is satisfied, if we choose either the constant $c_{2}$ with the opposite sign to $-d_{1} \omega_{(i)}(t)$ on $\bar{J}_{1} \cap \bar{J}_{2}$, or the constant $d_{2}$ with the opposite sign to $c_{1} \omega_{(i)}(t)$ on $\bar{J}_{1} \cap \bar{J}_{2}$.

In Case I-(iv), we have

$$
\begin{aligned}
\operatorname{Re} \omega(t) f(t) & =\omega_{(r)}(t) f_{(r)}(t)-\omega_{(\imath)}(t) f_{(i)}(t) \\
& =\left\{c_{1} \omega_{(r)}(t)-d_{1} \omega_{(\imath)}(t)\right\} e\left(t ; \gamma_{1}, \delta_{1}\right) \text { on } \bar{J}_{1} \cap \bar{J}_{2} ; \\
\operatorname{Im} \omega(t) f(t) & =\omega_{(r)}(t) f_{(i)}(t)+\omega_{(i)}(t) f_{(r)}(t) \\
& =\left\{d_{1} \omega_{(r)}(t)+c_{1} \omega_{(i)}(t)\right\} e\left(t ; \gamma_{1}, \delta_{1}\right) \text { on } \bar{J}_{1} \cap \bar{J}_{2} .
\end{aligned}
$$

Since 
ON THE CONSTRUCTION OF LINEARLY INDEPENDENT VECTORS

$$
\begin{array}{ll}
\left\{c_{1} \omega_{(r)}(t)\right\} \cdot\left\{-d_{1} \omega_{(i)}(t)\right\}=-c_{1} d_{1} \omega_{(r)}(t) \omega_{(i)}(t) \neq 0 & \text { on } \bar{J}_{1} \cap \bar{J}_{2} ; \\
\left\{d_{1} \omega_{(r)}(t)\right\} \cdot\left\{c_{1} \omega_{(i)}(t)\right\}=c_{1} d_{1} \omega_{r}(t) \omega_{(i)}(t) \neq 0 & \text { on } \bar{J}_{1} \cap \bar{J}_{2},
\end{array}
$$

one of these two products has the positive sign. Therefore the two factors $c_{1} \omega_{(r)}(t)$ and $-d_{1} \omega_{(i)}(t)$, or $d_{1} \omega_{(r)}(t)$ and $c_{1} \omega_{(i)}(t)$ in the above product which has the positive sign, have the same sign as each other on $\bar{J}_{1} \cap \bar{J}_{2}$. Hence, one of $\operatorname{Re} \omega(t) f(t)$ and $\operatorname{Im} \omega(t) f(t)$ has the definite sign on $\bar{J}_{1} \cap \bar{J}_{2}$.

If $\operatorname{Re} \omega(t) f(t)$ has the definite sign on $\bar{J}_{1} \cap \bar{J}_{2}$, then we choose the constant $c_{2}$ with the opposite sign to $\operatorname{Re} \omega(t) f(t)$ on $\bar{J}_{1} \cap \bar{J}_{2}$. If $\operatorname{Im} \omega(t) f(t)$ has the definite sign on $\bar{J}_{1} \cap \bar{J}_{2}$, then we choose the constant $d_{2}$ with the opposite sign to $\operatorname{Im} \omega(t) f(t)$ on $\bar{J}_{1} \cap \vec{J}_{2}$.

The procedure stated above, means that by choosing suitably one of the constants $c_{2}$ and $d_{2}$ in all cases, we can make the condition (2.1) be satisfied.

Next, under the circumstances that the condition (2.1) has been satisfied by determining suitably one of the constants $c_{2}$ and $d_{2}$, we shall show that we can choose the other of them so that the condition (2.2) is satisfied.

Since $f(t) \neq 0$ on the interval $J_{1}=\left(\gamma_{1}, \delta_{1}\right)$ and $\theta(t) g(t) \equiv 0$ on the interval $\left(\gamma_{1}, \gamma_{2}\right]$ for all non-zero values of $c_{2}$ and $d_{2}$, we have only to determine the non-zero constants $c_{2}$ and $d_{2}$, so that the condition $(2,2)$ is satisfied on the interval $J_{1} \cap J_{2}=\left(\gamma_{2}, \delta_{1}\right)$ instead of $J_{1}$.

For the accomplishment of our purpose, we shall distinguish four cases, according to the values of $\theta_{(r)}(t)$ and $\theta_{(i)}(t)$ on $\bar{J}_{1} \cap \bar{J}_{2}$ :

Case II- ( i ) $\quad \theta(t) \equiv 0 \quad$ on $\bar{J}_{1} \cap \bar{J}_{2}$,

Case II-(ii) $\quad \theta_{(r)}(t) \neq 0$ and $\theta_{(i)}(t) \equiv 0 \quad$ on $\bar{J}_{1} \cap \bar{J}_{2}$,

Case II-(iii) $\quad \theta_{(r)}(t) \equiv 0$ and $\theta_{(i)}(t) \neq 0$ on $\bar{J}_{1} \cap \bar{J}_{2}$,

Case II-(iv) $\theta_{(r)}(t) \neq 0$ and $\theta_{(i)}(t) \neq 0$ on $\bar{J}_{1} \cap \bar{J}_{2}$.

In Case II-(i), we have $\theta(t) g(t) \equiv 0$ on $\bar{J}_{1} \cap \bar{J}_{2}$ for all non-zero values of $c_{2}$ and $d_{2}$, and further $f(t) \neq 0$ on $J_{1}$. Hence the condition (2.2) is satisfied for all non-zero values of $c_{2}$ and $d_{2}$.

In Case II-(ii), we have

$$
\begin{array}{ll}
\operatorname{Re} \theta(t) g(t)=\theta_{(r)}(t) g_{(r)}(t)=c_{2} \theta_{(r)}(t) e\left(t ; \gamma_{2}, \delta_{2}\right) & \text { on } \bar{J}_{1} \cap \tilde{J}_{2} ; \\
\operatorname{Im} \theta(t) g(t)=\theta_{(r)}(t) g_{(i)}(t)=d_{2} \theta_{(r)}(t) e\left(t ; \gamma_{2}, \delta_{2}\right) & \text { on } \bar{J}_{1} \cap \tilde{J}_{2} .
\end{array}
$$

Although one of the constants $c_{2}$ and $d_{2}$ is already fixed in Cases I-(i) I-(iv), if we choose the other of them so that either

or

$$
\text { " } c_{2} \theta_{(r)}(t) \text { has the opposite sign to } c_{1} \text { on } \bar{J}_{1} \cap \bar{J}_{2} \text { ", }
$$

$$
\text { " } d_{2} \theta_{(r)}(t) \text { has the opposite sign to } d_{1} \text { on } \bar{J}_{1} \cap \bar{J}_{2} \text { ", }
$$

then the condition (2.2) is satisfied. 
In Case II-(iii), since

$$
\begin{array}{ll}
\operatorname{Re} \theta(t) g(t)=-\theta_{(i)}(t) g_{(i)}(t)=-d_{2} \theta_{(i)}(t) e\left(t ; \gamma_{2}, \delta_{2}\right) & \text { on } \bar{J}_{1} \cap \bar{J}_{2} ; \\
\operatorname{Im} \theta(t) g(t)=\theta_{(i)}(t) g_{(r)}(t)=c_{2} \theta_{(i)}(t) e\left(t ; \gamma_{2}, \delta_{2}\right) & \text { on } \bar{J}_{1} \cap \bar{J}_{2},
\end{array}
$$

we have only to determine one of the constants $c_{2}$ and $d_{2}$ so that either

or

$$
\text { " }-d_{2} \theta_{(i)}(t) \text { has the opposite sign to } c_{1} \text { on } \bar{J}_{1} \cap \bar{J}_{2} \text { ", }
$$

$$
\text { " } c_{2} \theta_{(i)}(t) \text { has the opposite sign to } d_{1} \text { on } \bar{J}_{1} \cap \bar{J}_{2} \text { ". }
$$

In Case II-(iv), we have

$$
\begin{aligned}
\operatorname{Re} \theta(t) g(t) & =\theta_{(r)}(t) g_{(r)}(t)-\theta_{(i)}(t) g_{(i)}(t) \\
& =\left\{c_{2} \theta_{(r)}(t)-d_{2} \theta_{(i)}(t)\right\} e\left(t ; \gamma_{2}, \delta_{2}\right) ; \\
\operatorname{Im} \theta(t) g(t) & =\theta_{(r)}(t) g_{(i)}(t)+\theta_{(i)}(t) g_{(r)}(t) \\
& =\left\{d_{2} \theta_{(r)}(t)+c_{2} \theta_{(i)}(t)\right\} e\left(t ; \gamma_{2}, \delta_{2}\right) .
\end{aligned}
$$

Although one of the constants $c_{2}$ and $d_{2}$ is already fixed in Cases I-(i) I-(iv), we can choose the other of them so that either

or

$$
\text { " } c_{2} \theta_{(r)}(t)-d_{2} \theta_{(i)}(t) \text { has the opposite sign to } c_{1} \text { on } \bar{J}_{1} \cap \bar{J}_{2} \text { ", }
$$

$$
\text { " } d_{2} \theta_{(r)}(t)+c_{2} \theta_{(i)}(t) \text { has the opposite sign to } d_{1} \text { on } \bar{J}_{1} \cap \bar{J}_{2} \text { ". }
$$

By means of this choice, the condition (2.2) is satisfied.

Thus this lemma has been completely proved.

Remark 1. Replacing $f(t)=\left(c_{1}+i d_{1}\right) e\left(t ; \gamma_{1}, \delta_{1}\right)$ by

$$
f(t)=\left(c_{1}+i d_{1}\right) e_{-}\left(t ; \delta_{1}\right),
$$

we obtain a result similar to Lemma 2 .

Remark 2. Replacing $g(t)=\left(c_{2}+i d_{2}\right) e\left(t ; \gamma_{2}, \grave{o}_{2}\right)$ by

$$
g(t)=\left(c_{2}+i d_{2}\right) e_{+}\left(t ; \gamma_{2}\right),
$$

we obtain a result similar to Lemma 2 .

\section{§3. Proof of Theorem 1 .}

We can form, by assumption, a set $\left\{J_{c}\right\}_{c}^{\ell_{l}^{0}=1}$ of intervals possessing the following properties: 
(i) $\bigcup_{c=1}^{e_{0}} J_{c}=J$;

(ii) $J_{1}=\left[\gamma_{1}, \delta_{1}\right), J_{\iota_{0}}=\left(\gamma_{\iota_{0}}, \delta_{\iota_{0}}\right], \gamma_{1}=\gamma, \delta_{\iota_{0}}=\delta, J_{\iota}=\left(\gamma_{\iota}, \delta_{\iota}\right)\left(\iota=2,3, \cdots, \iota_{0}-1\right)$;

(iii) $J_{\iota} \cap J_{\iota+1} \neq \varnothing\left(\iota=1,2, \cdots, \iota_{0}-1\right), J_{\iota} \cap J_{\iota^{\prime}}=\varnothing\left(\iota+1<\iota^{\prime}, \iota=1,2, \cdots, \iota_{0}-1\right)$, that is, $\gamma_{1}<\gamma_{2}<\delta_{1}<\cdots<\gamma_{\iota}<\delta_{\iota-1}<\gamma_{\iota+1}<\delta_{\iota}<\cdots<\delta_{\iota_{0}-2}<\gamma_{\iota_{0}}<\delta_{\iota_{0}-1}<\delta_{\iota_{0}}$ $\left(\iota=2,3, \cdots, \iota_{0}-1\right)$;

(iv) For each $J_{\iota}$, there exists a minor of degree $h$ of $X(t)$ which does not vanish on $\bar{J}_{\iota}$.

We consider first the intervals $J_{1}$ and $J_{2}$, and choose two minors $X\left(\begin{array}{cccc}k_{1} & k_{2} & \cdots & k_{h} \\ 1 & 2 & \cdots & h\end{array}\right)$ and $X\left(\begin{array}{cccc}m_{1} & m_{2} & \cdots & m_{h} \\ 1 & 2 & \cdots & h\end{array}\right)$ of degree $h$ of $X(t)$ such that a condition

$$
X\left(\begin{array}{cccc}
k_{1} & k_{2} & \cdots & k_{h} \\
1 & 2 & \cdots & h
\end{array}\right) \neq 0
$$

is satisfied on $\bar{J}_{1}$ and a condition

$$
X\left(\begin{array}{cccc}
m_{1} & m_{2} & \cdots & m_{h} \\
1 & 2 & \cdots & h
\end{array}\right) \neq 0
$$

is satisfied on $\bar{J}_{2}$.

We define an $(n-h)$-tuple $\left(k_{h+1}^{\prime}, k_{h+2}^{\prime}, \cdots, k_{n}^{\prime}\right)$ for $1 \leqq k_{1}<k_{2}<\cdots<k_{h} \leqq n$ in such a way that $1 \leqq k_{h+1}^{\prime}<k_{h+2}^{\prime}<\cdots<k_{n}^{\prime} \leqq n$ and $\left\{k_{1}, \cdots, k_{h}, k_{h+1}^{\prime}, \cdots, k_{n}^{\prime}\right\}=$ $\{1,2, \cdots, n\}$. That is, $k_{1}<k_{2}<\cdots<k_{n}$ and $k_{h+1}^{\prime}<k_{n+2}^{\prime}<\cdots<k_{n}^{\prime}$ form a complete system of indices $\{1,2, \cdots, n\}$. An $(n-h)$-tuple $\left(m_{h+1}^{\prime}, m_{h+2}^{\prime}, \cdots, m_{n}^{\prime}\right)$ is also defined for $1 \leqq m_{1}<m_{2}<\cdots<m_{n} \leqq n$ in the same manner.

We put

$$
\begin{array}{ll}
\hat{\boldsymbol{x}}_{k_{\rho}}(t)=\left(x_{k_{\rho^{1}}}(t), x_{k_{\rho^{2}}}(t), \cdots, x_{k_{\rho} h}(t)\right) & (\rho=1,2, \cdots, h), \\
\hat{\boldsymbol{x}}_{k_{\sigma}^{\prime}}(t)=\left(x_{k_{\sigma^{1}}}(t), x_{k_{\sigma^{\prime}}}(t), \cdots, x_{k_{\sigma^{\prime}} h}(t)\right) & (\sigma=h+1, h+2, \cdots, n), \\
\hat{\boldsymbol{x}}_{m_{\rho}}(t)=\left(x_{m_{\rho^{1}}}(t), x_{m_{\rho^{2}}}(t), \cdots, x_{m_{\rho^{\prime}}}(t)\right) & (\rho=1,2, \cdots, h), \\
\hat{\boldsymbol{x}}_{m_{\sigma}^{\prime}}(t)=\left(x_{m_{\sigma^{1}}}(t), x_{m_{\sigma^{2}}}(t), \cdots, x_{m_{\sigma^{\prime}} h}(t)\right) & (\sigma=h+1, h+2, \cdots, n) .
\end{array}
$$

Then it follows from the conditions (3.1) and (3.2), that there exist functions $\theta_{\sigma \rho}(t)(\rho=1,2, \cdots, h ; \sigma=h+1, h+2, \cdots, n)$ belonging to $K\left(\bar{J}_{1}\right)$ and functions $\omega_{\sigma \rho}(t)(\rho=1,2, \cdots, h ; \sigma=h+1, h+2, \cdots, n)$ belonging to $K\left(\bar{J}_{2}\right)$, such that

$$
\hat{\boldsymbol{x}}_{k_{\sigma}^{\prime}}(t)=\sum_{\rho=1}^{h} \theta_{\sigma \rho}(t) \hat{\boldsymbol{x}}_{k_{\rho}}(t) \quad(\sigma=h+1, h+2, \cdots, n) \quad \text { on } \bar{J}_{1},
$$

and

$$
\hat{\boldsymbol{x}}_{m_{\boldsymbol{\sigma}}^{\prime}}(t)=\sum_{\rho=1}^{h} \omega_{\sigma \rho}(t) \hat{\boldsymbol{x}}_{m_{\rho}}(t) \quad(\sigma=h+1, h+2, \cdots, n) \quad \text { on } \bar{J}_{2}
$$

The first step. 
We determine a vector $\boldsymbol{y}(t)=\operatorname{col}\left(y_{1}(t), y_{2}(t), \cdots, y_{n}(t)\right)$ on $\bar{J}_{1}$ in the following manner.

Concerning the component $y_{k_{h+1}^{\prime}}(t)$, we put

$$
y_{k_{h+1}^{\prime}}(t)=\left(c_{1}+i d_{1}\right) e_{-}\left(t ; \delta_{1}\right) ; \quad i=\sqrt{-1},
$$

where $c_{1}$ and $d_{1}$ are arbitrary real non-zero constants. As a matter of fact, it suffices for our present purpose that at least, any one of the constants $c_{1}$ and $d_{1}$ is not equal to zero. However, we take the constants $c_{1}$ and $d_{1}$ which are both non-zero for the sake of generality.

Concerning the other components of $\boldsymbol{y}(t)$, we put

$$
y_{k_{\rho}}(t) \equiv 0 \text { on } J(\rho=1, \cdots, h) \text { and } y_{k_{\sigma}^{\prime}}(t) \equiv 0 \text { on } J(\sigma=h+2, \cdots, n) \text {. }
$$

Then, in virtue of the fact that $y_{k_{h+1}^{\prime}}(t) \neq 0$ on $J_{1}$ and the condition (3.1) is satisfied on $\bar{J}_{1}$, we see

$$
\operatorname{rank} \boldsymbol{y}(t)=1 \text { on } J_{1} \text { and } \operatorname{rank}(X(t), \boldsymbol{y}(t))=h+1 \text { on } J_{1} \text {. }
$$

The second step.

We shall next construct a vector $\boldsymbol{y}(t)$, so that we have

$$
\begin{cases}\operatorname{rank} \boldsymbol{y}(t)=1 & \text { on } J_{1} \cup J_{2} ; \\ \operatorname{rank}(X(t), \boldsymbol{y}(t))=h+1 & \text { on } J_{1} \cup J_{2} .\end{cases}
$$

For the construction of $\boldsymbol{y}(t)$ on $J_{1} \cup J_{2}$, we shall distinguish three cases, according to the relation between the indices $\left(k_{1}, \cdots, k_{h}, k_{h+1}^{\prime}, \cdots, k_{n}^{\prime}\right)$ and $\left(m_{1}, \cdots, m_{h}, m_{h+1}^{\prime}, \cdots, m_{n}^{\prime}\right)$ :

Case S-(i) There exists an index $\sigma(1)$ such that $h+1 \leqq \sigma(1) \leqq n, m_{\sigma(1)}^{\prime}=k_{h+1}^{\prime}$.

Case S-(ii) There exist two indices $\rho(1)$ and $\sigma(2)$ such that

$$
1 \leqq \rho(1) \leqq h, m_{\rho(1)}=k_{h+1}^{\prime} \text { and } h+1 \leqq \sigma(2) \leqq n, m_{h+1}^{\prime}=k_{\sigma(2)}^{\prime} \text {. }
$$

Case S-(iii) There exist two indices $\rho(1)$ and $\rho(2)$ such that

$$
1 \leqq \rho(1) \leqq h, m_{\rho(1)}=k_{h+1}^{\prime} \text { and } 1 \leqq \rho(2) \leqq h, m_{h+1}^{\prime}=k_{\rho(2)} \text {. }
$$

In Case S-(i), we modify the component $y_{m_{\sigma(1)}^{\prime}}(t)\left(\equiv y_{k_{h+1}^{\prime}}(t)\right)$ determined at the first step, in the following way:

$$
y_{m_{\sigma(1)}^{\prime}}(t)\left(\equiv y_{k_{h+1}^{\prime}}(t)\right)=\left(c_{1}+i d_{1}\right) e_{-}\left(t ; \delta_{2}\right),
$$

and we leave the other components of $\boldsymbol{y}(t)$ as they are.

Then we have the condition (3.5).

In Cases S-(ii) and S-(iii), we must treat the function $\theta_{h+1, \rho(2)}(t)$ which appears in the relation (3.3), and the function $\omega_{h+1, \rho(1)}(t)$ which appears in the relation (3.4).

If we put

$$
\begin{array}{ll}
\theta_{h+1, \rho(2)}(t)=\varphi_{1}(t)+i \phi_{1}(t) ; & \varphi_{1}(t), \phi_{1}(t) \in C^{\mu}\left(\bar{J}_{1} ; \mathbf{R}\right), \\
\omega_{h+1, \rho(1)}(t)=\varphi_{2}(t)+i \phi_{2}(t) ; & \varphi_{2}(t), \phi_{2}(t) \in C^{\mu}\left(\bar{J}_{2} ; \mathbf{R}\right),
\end{array}
$$


then, in virtue of Lemma 1 , we can choose a closed subinterval $\left[\gamma_{2}^{*}, \delta_{1}^{*}\right]$ of the interval $\left[\gamma_{2}, \delta_{1}\right]$, such that each of the functions $\varphi_{1}(t), \phi_{1}(t), \varphi_{2}(t)$ and $\phi_{2}(t)$ is one-signed or identically equal to zero on the interval $\left[\gamma_{2}^{*}, \delta_{1}^{*}\right]$.

Replacing $\gamma_{2}$ and $\delta_{1}$ by $\gamma_{2}^{*}$ and $\delta_{1}^{*}$, we can assume, without loss of generality, that each of the functions $\varphi_{1}(t), \phi_{1}(t), \varphi_{2}(t)$ and $\phi_{2}(t)$ is one-signed or identically equal to zero on the interval $\left[\gamma_{2}, \delta_{1}\right]$. On this occasion, we must modify additionally the functions $e_{-}\left(t ; \delta_{1}\right)$ and $e\left(t ; \gamma_{2}, \delta_{2}\right)$.

In Case $\mathrm{S}$-(ii), we can determine, in virtue of Lemma 2 , two real non-zero constants $c_{2}$ and $d_{2}$ so that the function

$$
y_{m_{h+1}^{\prime}}(t)=\left(c_{2}+i d_{2}\right) e\left(t ; \gamma_{2}, \delta_{2}\right)
$$

satisfies a condition

$$
y_{m_{h+1}^{\prime}}(t)-\omega_{h+1, o(1)}(t) y_{k_{h+1}^{\prime}}(t) \neq 0 \text { on } J_{2} .
$$

Concerning the other components of $\boldsymbol{y}(t)$, we put

$$
\begin{array}{ll}
y_{m_{\rho}}(t) \equiv 0 & \text { on } J-J_{1}(\rho=1,2, \cdots, h), \\
y_{m_{\sigma}^{\prime}}(t) \equiv 0 & \text { on } J-J_{1}(\sigma=h+2, \cdots, n) .
\end{array}
$$

Then we can verify that the condition (3.5) is satisfied, in the following way: By the same reasoning as in the first step, we first obtain

$$
\operatorname{rank} \boldsymbol{y}(t)=1 \text { on } J_{1} \text { and } \operatorname{rank}(X(t), \boldsymbol{y}(t))=h+1 \text { on } J_{1} \text {. }
$$

We next consider the vector $\boldsymbol{y}(t)$ and the matrix $(X(t), \boldsymbol{y}(t))$ on the interval $J_{2}$.

We easily get rank $\boldsymbol{y}(t)=1$ on $J_{2}$, in virtue of the fact that $y_{m_{h+1}^{\prime}}(t) \neq 0$ on $J_{2}$.

Furthermore, making use of the relation (3.4) and the condition (3.6), and putting

$$
\tilde{y}_{m_{h+1}^{\prime}}(t)=y_{m_{h+1}^{\prime}}(t)-\omega_{h+1, \rho(1)}(t) y_{k_{h+1}^{\prime}}(t)
$$

we can transform the matrix $(X(t), \boldsymbol{y}(t))$ on $J_{2}$, by means of elementary operations, in the following manner:

$$
(X(t), \boldsymbol{y}(t)) \longrightarrow\left(\begin{array}{cc}
\hat{\boldsymbol{x}}_{m_{1}}(t) & 0 \\
\vdots & \vdots \\
\hat{\boldsymbol{x}}_{m_{\rho(1)}}(t) & y_{m_{\rho(1)}}(t) \\
\vdots & \vdots \\
\hat{\boldsymbol{x}}_{m_{h}}(t) & 0 \\
\hat{\boldsymbol{x}}_{m_{h+1}^{\prime}}(t) & y_{m_{h+1}^{\prime}}(t) \\
\vdots & 0 \\
\vdots & \vdots \\
\hat{\boldsymbol{x}}_{m_{h}^{\prime}}(t) & 0
\end{array}\right)
$$




$$
\longrightarrow\left(\begin{array}{cc}
\hat{\boldsymbol{x}}_{m_{1}}(t) & 0 \\
\vdots & \vdots \\
\hat{\boldsymbol{x}}_{m_{\rho(1)}}(t) & y_{m \rho(1)}(t) \\
\vdots & \vdots \\
\hat{\boldsymbol{x}}_{m_{h}}(t) & 0 \\
\boldsymbol{o} & \tilde{y}_{m_{h+1}^{\prime}}(t) \\
\boldsymbol{o} & -\omega_{h+2, \rho(1)}(t) y_{m \rho(1)}(t) \\
\vdots & \vdots \\
\boldsymbol{o} & -\omega_{n, \rho(1)}(t) y_{m \rho(1)}(t)
\end{array}\right) \rightarrow\left(\begin{array}{cc}
\hat{\boldsymbol{x}}_{m_{1}}(t) & 0 \\
\vdots & \vdots \\
\hat{\boldsymbol{x}}_{m_{\rho(1)}}(t) & \vdots \\
\vdots & \vdots \\
\hat{\boldsymbol{x}}_{m_{h}}(t) & 0 \\
\boldsymbol{o} & \tilde{y}_{m_{h+1}^{\prime}}(t) \\
\boldsymbol{o} & 0 \\
\vdots & \vdots \\
\boldsymbol{o} & 0
\end{array}\right) .
$$

Therefore we obtain the condition (3.5), in virtue of the condition (3.6).

In Case S-(iii), we can determine, in virtue of Lemma 2, two real non-zero constants $c_{2}$ and $d_{2}$ so that the function

$$
y_{m_{h+1}^{\prime}}(t)=\left(c_{2}+i d_{2}\right) e\left(t ; \gamma_{2}, \delta_{2}\right)
$$

satisfies a condition

$$
y_{k_{h+1}^{\prime}}(t)-\theta_{h+1, \rho(2)}(t) y_{m_{h+1}^{\prime}}(t) \neq 0 \quad \text { on } J_{1}
$$

and the condition (3.6).

Further we define the other components of $\boldsymbol{y}(t)$ in the same way as in Case S-(ii).

On this occasion, we can prove the condition

$$
\operatorname{rank} \boldsymbol{y}(t)=1 \text { on } J_{2} \text { and } \operatorname{rank}(X(t), \boldsymbol{y}(t))=h+1 \text { on } J_{2} \text {, }
$$

on the same lines as in Case S-(ii).

We wish next to verify that

$$
\operatorname{rank} \boldsymbol{y}(t)=1 \text { on } J_{1} \text { and } \operatorname{rank}(X(t), \boldsymbol{y}(t))=h+1 \text { on } J_{1} \text {. }
$$

We easily see $\operatorname{rank} \boldsymbol{y}(t)=1$ on $J_{1}$, because $y_{k_{h+1}^{\prime}}(t) \neq 0$ on $J_{1}$.

Moreover, taking the relation (3.3) and the condition (3.7) into account and putting

$$
\check{y}_{k_{h+1}^{\prime}}(t)=y_{k_{h+1}^{\prime}}(t)-\theta_{h+1, \rho(2)}(t) y_{m_{h+1}^{\prime}}(t),
$$

we can transform the matrix $(X(t), \boldsymbol{y}(t))$ on $J_{1}$, by means of elementary operations, in the following manner: 
ON THE CONSTRUCTION OF LINEARLY INDEPENDENT VECTORS

$(X(t), \boldsymbol{y}(t)) \longrightarrow\left(\begin{array}{cc}\hat{\boldsymbol{x}}_{k_{1}}(t) & 0 \\ \vdots & \vdots \\ \hat{\boldsymbol{x}}_{k_{\rho(2)}}(t) & y_{m_{h+1}^{\prime}}(t) \\ \vdots & \vdots \\ \hat{\boldsymbol{x}}_{k_{h}}(t) & 0 \\ \hat{\boldsymbol{x}}_{k_{h+1}^{\prime}}(t) & y_{k_{h+1}^{\prime}}(t) \\ \vdots & 0 \\ \vdots & \vdots \\ \hat{\boldsymbol{x}}_{k_{n}^{\prime}}(t) & 0\end{array}\right)$

$\longrightarrow\left(\begin{array}{cc}\hat{\boldsymbol{x}}_{k_{1}}(t) & 0 \\ \vdots & \vdots \\ \hat{\boldsymbol{x}}_{k_{\rho(2)}}(t) & y_{m_{h+1}^{\prime}}(t) \\ \vdots & \vdots \\ \hat{\boldsymbol{x}}_{k_{h}}(t) & 0 \\ \boldsymbol{o} & \check{y}_{k_{h+1}^{\prime}}(t) \\ \boldsymbol{o} & -\theta_{h+2, \rho(2)} y_{m_{h+1}^{\prime}}(t) \\ \vdots & \vdots \\ \boldsymbol{o} & -\theta_{n, \rho(2)} y_{m_{h+1}^{\prime}}(t)\end{array}\right) \longrightarrow\left(\begin{array}{cc}\hat{\boldsymbol{x}}_{k_{1}}(t) & 0 \\ \vdots & \vdots \\ \hat{\boldsymbol{x}}_{k_{\rho(2)}}(t) & \vdots \\ \vdots & \vdots \\ \hat{\boldsymbol{x}}_{k_{h}}(t) & 0 \\ \boldsymbol{o} & \check{y}_{k_{h+1}^{\prime}}(t) \\ \vdots & 0 \\ \vdots & \vdots \\ \boldsymbol{o} & 0\end{array}\right)$.

Hence we obtain the condition (3.5), in virtue of the condition (3.7).

By repeating the process employed above for each pair $\left\{J_{\iota}, J_{c+1}\right\}$ $\left(\iota=1,2, \cdots, \iota_{0}-1\right)$ of intervals, we get the desired vector $\boldsymbol{y}(t)=\operatorname{col}\left(y_{1}(t), y_{2}(t), \cdots\right.$, $\left.y_{n}(t)\right)$ satisfying the condition (1.3).

In the accomplishment of this proof, we must examine which of Casess S-(i) $\sim \mathrm{S}$-(iii) occurs, and if necessary, we choose the interval $\left[\delta_{c+1}^{*}, \gamma_{c}^{*}\right]$ corresponding to the interval $\left[\delta_{2}^{*}, \gamma_{1}^{*}\right]$ taken at the begining of the consideration for Cases S-(ii) and S-(iii), and we must adopt $\delta_{\iota+1}^{*}$ and $\gamma_{\iota}^{*}$ anew for $\delta_{\iota+1}$ and $\gamma_{\iota}$.

Furthermore we use the functions $e\left(t ; \gamma_{\iota}, \delta_{\iota}\right)$ for $J_{\iota}\left(\iota=2,3, \cdots, \iota_{0}-1\right), e_{-}\left(t ; \delta_{1}\right)$ for $J_{1}$ and $e_{+}\left(t, \gamma_{\iota_{0}}\right)$ for $J_{c_{0}}$.

\section{$\S 4$. Summary about solutions of a linear matrix equation.}

In this section, we shall summarize the matters which are used for the proof of Theorem 3.

Let $I_{1}$ and $I_{2}$ be two intervals such that $I_{1}=\left[\alpha_{1}, \beta_{1}\right)$ or $I_{1}=\left(\alpha_{1}, \beta_{1}\right)$, and $I_{2}=\left(\alpha_{2}, \beta_{2}\right)$ or $I_{2}=\left(\alpha_{2}, \beta_{2}\right]$ and further $\alpha_{1}<\alpha_{2}<\beta_{1}<\beta_{2}$.

Let $B(t)$ be the square matrix of degree $n$, which is given in $\S 1$. Assume that for a positive integer $s: 2 \leqq s \leqq n-1$, the condition (1.7) is satisfied on $\bar{I}_{1} \cup \bar{I}_{2}$ 
and further that a condition

$$
B\left(\begin{array}{cccc}
j_{1} & j_{2} & \cdots & j_{r} \\
k_{1} & k_{2} & \cdots & k_{r}
\end{array}\right) \neq 0
$$

is satisfied on $\bar{I}_{1}$ and a condition

$$
B\left(\begin{array}{cccc}
l_{1} & l_{2} & \cdots & l_{r} \\
m_{1} & m_{2} & \cdots & m_{r}
\end{array}\right) \neq 0
$$

is satisfied on $\bar{I}_{2}$.

We define an $(n-r)$-tuple $\left(k_{r+1}^{\prime}, k_{r+2}^{\prime}, \cdots, k_{n}^{\prime}\right)$ for $1 \leqq k_{1}<k_{2}<\cdots<k_{r} \leqq n$ in such a way that $1 \leqq k_{r+1}^{\prime}<k_{r+2}^{\prime}<\cdots<k_{n}^{\prime} \leqq n$ and $\left\{k_{1}, \cdots, k_{r}, k_{r+1}^{\prime}, \cdots, k_{n}^{\prime}\right\}=$ $\{1,2, \cdots, n\}$. An $(n-r)$-tuple $\left(m_{r+1}^{\prime}, m_{r+2}^{\prime}, \cdots, m_{n}^{\prime}\right)$ is also defined for $1 \leqq m_{1}<m_{2}$ $<\cdots<m_{r} \leqq n$ in the same way.

Let us consider an $n \times s_{1}$ matrix $\left(1 \leqq s_{1} \leqq s\right) P(t)$ whose components all belong to $K\left(\bar{I}_{1}\right)$ :

and put

$$
P(i)=\left(\begin{array}{cccc}
p_{11}(t) & p_{12}(t) & \cdots & p_{1 s_{1}}(t) \\
p_{21}(t) & p_{22}(t) & \cdots & p_{2 s_{1}}(t) \\
\vdots & \vdots & & \vdots \\
p_{n 1}(t) & p_{n 2}(t) & \cdots & p_{n s_{1}}(t)
\end{array}\right)
$$

$$
\begin{aligned}
& \hat{\boldsymbol{p}}_{k_{\rho}}(t)=\left(p_{k_{\rho} 1}(t), p_{k_{\rho}{ }^{2}}(t), \cdots, p_{k_{\rho} s_{1}}(t)\right) \quad(\rho=1,2, \cdots, r) \text {; } \\
& \hat{\boldsymbol{p}}_{k^{\prime}}(t)=\left(p_{k^{\prime}{ }^{1}}(t), p_{k^{\prime}{ }^{2}}(t), \cdots, p_{k^{\prime} s^{\prime}}(t)\right) \quad(\sigma=r+1, r+2, \cdots, n) \text {. }
\end{aligned}
$$

Then, in virtue of Cramer's rule, we recall the following fact.

The matrix $P(t)$ satisfies a linear equation

$$
B(t) P(t)=O
$$

on $\bar{I}_{1}$, if and only if the vectors $\hat{\boldsymbol{p}}_{k_{\rho}}(t)(\rho=1,2, \cdots, r)$ can be represented as linear combinations of the vectors $\hat{\boldsymbol{p}}_{k_{\sigma}^{\prime}}(t)(\sigma=r+1, r+2, \cdots, n)$ :

$$
\hat{\boldsymbol{p}}_{k_{\rho}}(t)=\sum_{\sigma=r+1}^{n} \boldsymbol{\xi}_{\rho \sigma}(t) \hat{\boldsymbol{p}}_{k_{\sigma}^{\prime}}(t) \quad(\rho=1,2, \cdots, r)
$$

with coefficients $\xi_{\rho \sigma}(t)$ which belong to $K\left(\bar{I}_{1}\right)$ and are expressed by

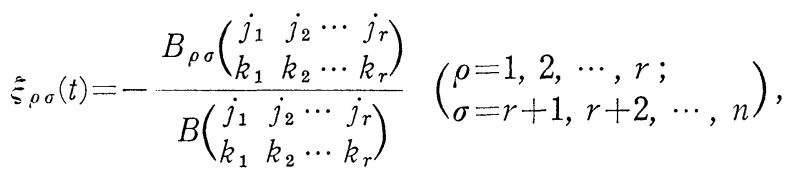

where

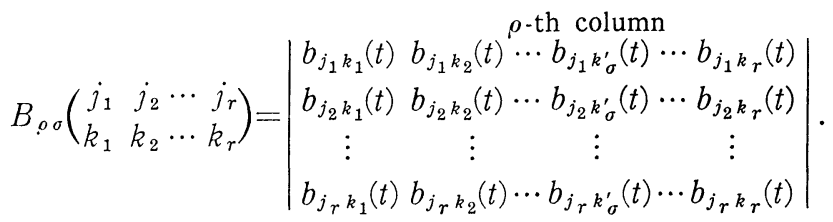


Therefore, we obtain the following proposition:

Proposition 1. Let $B(t)$ be the matrix given in $\S 1$ and let $P(t)$ be an $n \times s_{1}$ matrix $\left(1 \leqq s_{1} \leqq s\right)$, whose components all belong to $K\left(\bar{I}_{1}\right)$ and which satisfies the equation (4.3) on $\bar{I}_{1}$. Then a condition

$$
\operatorname{rank} P(t)=s_{1}
$$

is satisfied on $\bar{I}_{1}$, if and only if a condition

is satisfied on $\bar{I}_{1}$.

$$
\operatorname{rank}\left(\begin{array}{c}
\hat{\boldsymbol{p}}_{k_{r+1}^{\prime}}(t) \\
\vdots \\
\hat{\boldsymbol{p}}_{k_{n}^{\prime}}(t)
\end{array}\right)=s_{1}
$$
$K\left(\bar{I}_{2}\right)$ :

Let us next consider an $n \times s_{1}$ matrix $Q(t)$ whose components all belong to

and put

$$
Q(t)=\left(\begin{array}{cccc}
q_{11}(t) & q_{12}(t) & \cdots & q_{1 s_{1}}(t) \\
q_{21}(t) & q_{22}(t) & \cdots & q_{2 s_{1}}(t) \\
\vdots & \vdots & & \vdots \\
q_{n 1}(t) & q_{n 2}(t) & \cdots & q_{n s_{1}}(t)
\end{array}\right)
$$

$$
\begin{aligned}
& \hat{\boldsymbol{q}}_{m_{\rho}}(t)=\left(q_{m_{\rho^{1}}}(t), q_{m_{\rho^{2}}}(t), \cdots, q_{m_{\rho} s_{1}}(t)\right) \quad(\rho=1,2, \cdots, r), \\
& \hat{\boldsymbol{q}}_{m_{\sigma}^{\prime}}(t)=\left(q_{m_{\boldsymbol{\sigma}^{\prime}}}(t), q_{m_{\sigma^{2}}{ }^{2}}(t), \cdots, q_{m_{\boldsymbol{\sigma}^{\prime}}{ }^{\prime}}(t)\right) \quad(\sigma=r+1, r+2, \cdots, n) \text {. }
\end{aligned}
$$

Then, on the same ground as for $P(t)$, we know the following fact.

The matrix $Q(t)$ satisfies a linear equation

$$
B(t) Q(t)=O
$$

on $\bar{I}_{2}$, if and only if the vectors $\hat{\boldsymbol{q}}_{m \rho}(t)(\rho=1,2, \cdots, r)$ can be represented as linear combinations of the vectors $\hat{\boldsymbol{q}}_{m_{\sigma}^{\prime}}(t)(\sigma=r+1, r+2, \cdots, n)$ :

$$
\hat{\boldsymbol{q}}_{m_{\rho}}(t)=\sum_{\sigma=r+1}^{n} \eta_{\rho \sigma}(t) \hat{\boldsymbol{q}}_{m_{\sigma}^{\prime}}(t) \quad(\rho=1,2, \cdots, r)
$$

with coefficients $\eta_{\rho \sigma}(t)$ which belong to $K\left(\bar{I}_{2}\right)$ and are expressed by

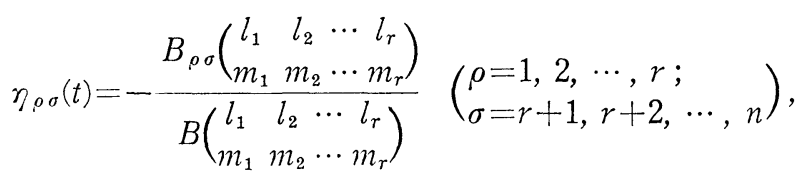

where 


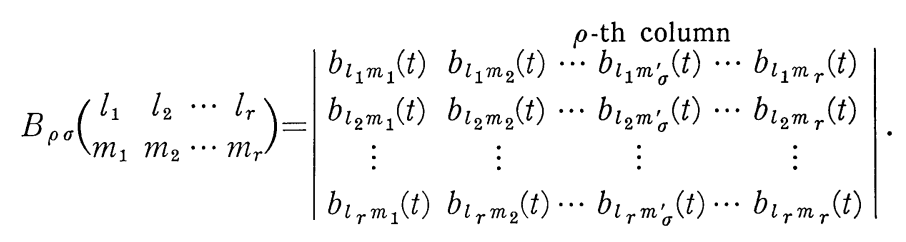

In this case, we get also, for $Q(t)$, a proposition similar to Proposition 1 .

Concerning the relation between the matrices $P(t)$ and $Q(t)$, we have the following lemma :

LEMMA 3. Let $B(t)$ be the matrix given in $\S 1$. Let $P(t)$ and $Q(t)$ be the $n \times s$ matrices-that is, $s_{1}=s-$, which are given above and satisfy the equation (4.3) on $\bar{I}_{1}$ and the equation (4.6) on $\bar{I}_{2}$ respectively. Suppose further that conditions

$$
\operatorname{rank} P(t)=s \text { and } \operatorname{rank} Q(t)=s
$$

are satisfied on $\bar{I}_{1}$ and on $\bar{I}_{2}$ respectively.

Then there exists a square matrix $C(t)$ of degree s such that

( I ) Every component of $C(t)$ belongs to $K\left(\bar{I}_{1} \cap \bar{I}_{2}\right)$;

(II) $\operatorname{rank} C(t)=s$ on $\bar{I}_{1} \cap \bar{I}_{2}$;

(III) $P(t)=Q(t) C(t)$ on $\bar{I}_{1} \cap \bar{I}_{2}$.

For the proof of this lemma, see the proof of Lemma 2 in the previous paper [1].

\section{$\S 5$. Proof of Theorem 3 .}

Let $X(t)$ denote the matrix :

$$
X(t)=\left(\boldsymbol{x}_{1}(t), \boldsymbol{x}_{2}(t), \cdots, \boldsymbol{x}_{s^{\prime}}(t)\right),
$$

where $\boldsymbol{x}_{k}(t)=\operatorname{col}\left(x_{1 k}(t), x_{2 k}(t), \cdots, x_{n k}(t)\right)\left(k=1,2, \cdots, s^{\prime}\right)$ are $s^{\prime}$ prescribed vectors belonging to $W(I)$.

Now, by assumption, we can choose a set $\left\{I_{\kappa}\right\}_{\kappa=1}^{\kappa_{0}}$ of intervals possessing the following properties:

(i) $I=\bigcup_{\kappa=1}^{\kappa_{0}} I_{\kappa}$;

(ii) $I_{1}=\left[\alpha_{1}, \beta_{1}\right), I_{\kappa_{0}}=\left(\alpha_{\kappa_{0}}, \beta_{\kappa_{0}}\right], \alpha_{1}=\alpha, \beta_{\kappa_{0}}=\beta$,

$I_{\kappa}=\left(\alpha_{\kappa}, \beta_{\kappa}\right)\left(\kappa=2,3, \cdots, \kappa_{0}-1\right)$;

(iii) $I_{\kappa} \cap I_{\kappa+1} \neq \varnothing\left(\kappa=1,2, \cdots, \kappa_{0}-1\right)$,

$I_{\kappa} \cap I_{\kappa^{\prime}}=\varnothing\left(\kappa+1<\kappa^{\prime}, \kappa=1,2, \cdots, \kappa_{0}-2\right)$,

that is, $\alpha_{1}<\alpha_{2}<\beta_{1}<\cdots<\alpha_{\kappa}<\beta_{\kappa-1}<\alpha_{\kappa+1}<\beta_{\kappa}<\cdots<\beta_{\kappa_{0}-2}<\alpha_{\kappa_{0}}<\beta_{\kappa_{0}-1}<\beta_{\kappa_{0}}$

$\left(\kappa=2,3, \cdots, \kappa_{0}-1\right)$;

(iv) For each $I_{k}$, there exists a minor of degree $r$ of $B(t)$ which does not vanish on the closure $\bar{I}_{\kappa}$ of $I_{\kappa}$. 
For the details about the existence of such a set $\left\{I_{k}\right\}_{k=1}^{\alpha_{0}}$ of intervals, see the proof of Theorem in the previous paper [1].

Let us assume that a condition

$$
B\left(\begin{array}{cccc}
j_{1} & j_{2} & \cdots & j_{r} \\
k_{1} & k_{2} & \cdots & k_{r}
\end{array}\right) \neq 0
$$

is satisfied on $\bar{I}_{1}$, and a condition

$$
B\left(\begin{array}{cccc}
l_{1} & l_{2} & \cdots & l_{r} \\
m_{1} & m_{2} & \cdots & m_{r}
\end{array}\right) \neq 0
$$

is satisfied on $\bar{I}_{2}$.

We here remark that we are able, without loss of generality, to take the closures $\bar{I}_{1}=\left[\alpha_{1}, \beta_{1}\right]$ and $\bar{I}_{2}=\left[\alpha_{2}, \beta_{2}\right]$ of $I_{1}$ and $I_{2}$, for the conditions (5.1) and (5.2), instead of $I_{1}$ and $I_{2}$ which were taken for the similar conditions in the proof of Theorem in the previous paper [1].

We put

$$
\begin{aligned}
& \hat{\boldsymbol{x}}_{k_{\rho}}(t)=\left(x_{k_{\rho^{1}}}(t), x_{k_{\rho^{2}}}(t), \cdots, x_{k_{\rho^{\prime}}}(t)\right) \quad(\rho=1,2, \cdots, r), \\
& \hat{\boldsymbol{x}}_{k_{\sigma}^{\prime}}(t)=\left(x_{k_{\sigma^{\prime}}{ }^{1}}(t), x_{k_{\sigma^{\prime}}{ }^{2}}(t), \cdots, x_{k_{\sigma^{\prime}} s^{\prime}}(t)\right) \quad(\sigma=r+1, r+2, \cdots, n) \text {. }
\end{aligned}
$$

Then, in virtue of the conditions (1.7) and (5.1), the vectors $\hat{\boldsymbol{x}}_{k_{\rho}}(t)$ $(\rho=1,2, \cdots, r)$ can be represented as combinations of the vectors $\hat{\boldsymbol{x}}_{k_{\sigma}^{\prime}}(t)$ $(\sigma=r+1, r+2, \cdots, n)$ :

$$
\hat{\boldsymbol{x}}_{k_{\rho}}(t)=\sum_{\sigma=r+1}^{n} \xi_{\rho \sigma}(t) \hat{\boldsymbol{x}}_{k_{\sigma}^{\prime}}(t) \quad(\rho=1,2, \cdots, r),
$$

where $\xi_{\rho \sigma}(t)$ are the same as in the linear combinations (4.4).

Furthermore, it follows from the condition (1.9) and Proposition 1 given in $\S 4$, that

$$
\operatorname{rank}\left(\begin{array}{c}
\hat{\boldsymbol{x}}_{k_{r+1}^{\prime}}(t) \\
\vdots \\
\hat{\boldsymbol{x}}_{k_{n}^{\prime}}(t)
\end{array}\right)=s^{\prime} \text { on } \bar{I}_{1} .
$$

The condition (5.4) and Theorem 2 imply, therefore, that there exist vectors $\hat{\boldsymbol{y}}_{k_{\sigma}}(t)(\sigma=r+1, r+2, \cdots, n)$ such that

$$
\begin{aligned}
& \hat{\boldsymbol{y}}_{k_{\sigma}^{\prime}}(t)=\left(y_{k_{\sigma}^{\prime} s^{\prime}+1}(t), y_{k_{\sigma}^{\prime} s^{\prime}+2}(t), \cdots, y_{k_{\sigma}^{\prime}}(t)\right) ; \\
& y_{k_{\sigma}^{\prime} g}(t) \in K\left(\bar{I}_{1}\right) \quad\left(g=s^{\prime}+1, s^{\prime}+2, \cdots, s ; \sigma=r+1, r+2, \cdots, n\right),
\end{aligned}
$$

and

$$
\operatorname{rank}\left(\begin{array}{c}
\hat{\boldsymbol{y}}_{k_{r+1}^{\prime}}(t) \\
\vdots \\
\hat{\boldsymbol{y}}_{k_{n}^{\prime}}(t)
\end{array}\right)=s-s^{\prime} \quad \text { on } \bar{I}_{1} \text {, }
$$


and

$$
\operatorname{rank}\left(\begin{array}{cc}
\hat{\boldsymbol{x}}_{k_{r+1}^{\prime}}(t) & \hat{\boldsymbol{y}}_{k_{r+1}^{\prime}}(t) \\
\vdots & \vdots \\
\hat{\boldsymbol{x}}_{k_{n}^{\prime}}(t) & \hat{\boldsymbol{y}}_{k_{n}^{\prime}}(t)
\end{array}\right)=s \text { on } \bar{I}_{1}
$$

Further, we define vectors

by means of

$$
\hat{\boldsymbol{y}}_{k_{\rho}}(t)=\left(y_{k_{\rho^{s^{\prime}+1}}}(t), y_{k_{\rho^{s^{\prime}+2}}}(t), \cdots, y_{k_{\rho}}(t)\right) \quad(\rho=1,2, \cdots, r)
$$

$$
\hat{\boldsymbol{y}}_{k_{\rho}}(t)=\sum_{\sigma=r+1}^{n} \boldsymbol{\xi}_{\rho \sigma}(t) \hat{\boldsymbol{y}}_{k^{\prime}}(t),
$$

where $\xi_{\rho \sigma}(t)$ are the same as in the linear combinations (4.4), and we put

$$
\begin{cases}\hat{\boldsymbol{p}}_{k_{\rho}}(t)=\left(\hat{\boldsymbol{x}}_{k_{\rho}}(t), \hat{\boldsymbol{y}}_{k_{\rho}}(t)\right) & (\rho=1,2, \cdots, r), \\ \hat{\boldsymbol{p}}_{k_{\sigma}^{\prime}}(t)=\left(\hat{\boldsymbol{x}}_{k_{\sigma}^{\prime}}(t), \hat{\boldsymbol{y}}_{k_{\sigma}^{\prime}}(t)\right) & (\sigma=r+1, r+2, \cdots, n) .\end{cases}
$$

Rearranging the rows of the matrix :

$$
\hat{P}(t)=\left(\begin{array}{c}
\hat{\boldsymbol{p}}_{k_{1}}(t) \\
\vdots \\
\hat{\boldsymbol{p}}_{k_{r}}(t) \\
\hat{\boldsymbol{p}}_{k_{r+1}^{\prime}}(t) \\
\vdots \\
\hat{\boldsymbol{p}}_{k_{n}^{\prime}}(t)
\end{array}\right)
$$

in the original order :

$$
\begin{aligned}
& P^{(1)}(t)=\left(\begin{array}{c}
\hat{\boldsymbol{p}}_{1}(t) \\
\vdots \\
\hat{\boldsymbol{p}}_{n}(t)
\end{array}\right)=(X(t), Y(t)) ; \\
& X(t)=\left(\begin{array}{c}
\hat{\boldsymbol{x}}_{1}(t) \\
\vdots \\
\hat{\boldsymbol{x}}_{n}(t)
\end{array}\right), \quad Y(t)=\left(\begin{array}{c}
\hat{\boldsymbol{y}}_{1}(t) \\
\vdots \\
\hat{\boldsymbol{y}}_{n}(t)
\end{array}\right),
\end{aligned}
$$

we have

$$
\operatorname{rank} P^{(1)}(t)=s \text { on } \bar{I}_{1} \text {, }
$$

and

$$
B(t) P^{(1)}(t)=O \quad \text { on } \bar{I}_{1} .
$$

Next we consider the vectors $\boldsymbol{x}_{1}(t), \boldsymbol{x}_{2}(t), \cdots, \boldsymbol{x}_{s^{\prime}}(t)$ on $\bar{I}_{2}$. If we put

$$
\hat{\boldsymbol{x}}_{m_{\rho}}(t)=\left(x_{m_{\rho^{1}}}(t), x_{m_{\rho^{2}}}(t), \cdots, x_{m_{\rho^{s^{\prime}}}}(t)\right) \quad(\rho=1,2, \cdots, r) ;
$$




$$
\hat{\boldsymbol{x}}_{m_{\sigma}^{\prime}}(t)=\left(x_{m_{\sigma^{1}}{ }^{1}}(t), x_{m_{\sigma^{\prime}}{ }^{2}}(t), \cdots, x_{m_{\sigma^{\prime}}{ }^{\prime}}(t)\right) \quad(\sigma=r-1, r+2, \cdots, n),
$$

then, in virtue of the condition (5.2), the vectors $\hat{\boldsymbol{x}}_{m_{\rho}}(t)(\rho=1,2, \cdots, r)$ can be represented as linear combinations of the vectors $\hat{\boldsymbol{x}}_{m_{\sigma}^{\prime}}(t)(\boldsymbol{\sigma}=r+1, r+2, \cdots, n)$ :

$$
\hat{\boldsymbol{x}}_{m_{\rho}}(t)=\sum_{\sigma=r+1}^{n} \eta_{\rho \sigma}(t) \hat{\boldsymbol{x}}_{m_{\sigma}^{\prime}}(t) \quad(\rho=1,2, \cdots, r),
$$

where $\eta_{o \sigma}(t)$ are the same as in the linear combinations (4.7).

By the same reasoning as for the condition (5.4), we have

$$
\operatorname{rank}\left(\begin{array}{c}
\hat{\boldsymbol{x}}_{m_{r+1}^{\prime}}(t) \\
\vdots \\
\hat{\boldsymbol{x}}_{m_{n}^{\prime}}(t)
\end{array}\right)=s^{\prime} \text { on } \bar{I}_{2} \text {. }
$$

Therefore, it follows from Theorem 2, that there exist vectors $\hat{\boldsymbol{z}}_{m_{\sigma}^{\prime}}(t)$ $(\sigma=r+1, r \div 2, \cdots, n)$ such that

and

$$
\begin{aligned}
& \hat{\boldsymbol{z}}_{m_{\sigma}^{\prime}}(t)=\left(z_{m_{\sigma} s^{\prime}+1}(t), z_{m_{\sigma^{\prime}} s^{\prime}+2}(t), \cdots, z_{m_{\sigma}^{\prime} s}(t)\right) ; \\
& z_{m_{\sigma}^{\prime} g}(t) \in K\left(\bar{I}_{2}\right) \quad\left(g=s^{\prime}+1, s^{\prime}+2, \cdots, s ; \sigma=r+1, r+2, \cdots, n\right),
\end{aligned}
$$

and

$$
\operatorname{rank}\left(\begin{array}{c}
\hat{\boldsymbol{z}}_{m_{r+1}^{\prime}}(t) \\
\vdots \\
\hat{\boldsymbol{z}}_{m_{n}^{\prime}}^{\prime}(t)
\end{array}\right)=s-s^{\prime} \text { on } \tilde{I}_{2}
$$

and

We define vectors

$$
\operatorname{rank}\left(\begin{array}{cc}
\hat{\boldsymbol{x}}_{m_{r+1}^{\prime}}(t) & \hat{\boldsymbol{z}}_{m_{r+1}^{\prime}}(t) \\
\vdots & \vdots \\
\hat{\boldsymbol{x}}_{m_{n}^{\prime}}^{\prime}(t) & \hat{\boldsymbol{z}}_{m_{n}^{\prime}}^{\prime}(t)
\end{array}\right)=s \text { on } \bar{I}_{2}
$$

$$
\hat{\boldsymbol{z}}_{m_{\rho}}(t)=\left(z_{m_{\rho^{s^{\prime}+1}}}(t), z_{m_{\rho^{s^{\prime}+2}}}(t), \cdots, z_{m_{\rho} \rho^{s}}(t)\right) \quad(\rho=1,2, \cdots, r)
$$

by means of

$$
\hat{\boldsymbol{z}}_{m_{\rho}}(t)=\sum_{\sigma=r+1}^{n} \eta_{\rho \sigma}(t) \hat{\boldsymbol{z}}_{m_{\sigma}^{\prime}}(t)
$$

where $\gamma_{\rho \sigma}(t)$ are the same as in the linear combinations $(4.7)$, and we put

$$
\begin{cases}\hat{\boldsymbol{q}}_{m}(t)=\left(\hat{\boldsymbol{x}}_{m_{\rho}}(t), \hat{\boldsymbol{z}}_{m_{\rho}}(t)\right) & (\rho=1,2, \cdots, r), \\ \hat{\boldsymbol{q}}_{m_{\sigma}^{\prime}}(t)=\left(\hat{\boldsymbol{x}}_{m_{\sigma}^{\prime}}(t), \hat{\boldsymbol{z}}_{m_{\boldsymbol{\sigma}}^{\prime}}(t)\right) & (\sigma=r+1, r \div 2, \cdots, n) .\end{cases}
$$

Rearranging the rows of the matrix : 


$$
\hat{Q}(t)=\left(\begin{array}{c}
\hat{\boldsymbol{q}}_{m_{1}}(t) \\
\vdots \\
\hat{\boldsymbol{q}}_{m_{r}}(t) \\
\hat{\boldsymbol{q}}_{m_{r+1}^{\prime}}(t) \\
\vdots \\
\hat{\boldsymbol{q}}_{m_{n}^{\prime}}(t)
\end{array}\right)
$$

in the original order :

we obtain

$$
\begin{aligned}
& Q(t)=\left(\begin{array}{c}
\hat{\boldsymbol{q}}_{1}(t) \\
\vdots \\
\hat{\boldsymbol{q}}_{n}(t)
\end{array}\right)=(X(t), Z(t)) ; \\
& X(t)=\left(\begin{array}{c}
\hat{\boldsymbol{x}}_{1}(t) \\
\vdots \\
\hat{\boldsymbol{x}}_{n}(t)
\end{array}\right), \quad Z(t)=\left(\begin{array}{c}
\hat{\boldsymbol{z}}_{1}(t) \\
\vdots \\
\hat{\boldsymbol{z}}_{n}(t)
\end{array}\right),
\end{aligned}
$$

$$
\operatorname{rank} Q(t)=s \text { on } \bar{I}_{2} \text {, }
$$

and

$$
B(t) Q(t)=O \quad \text { on } \bar{I}_{2} \text {. }
$$

In virtue of Lemma 3 given in $\S 4$, there exists a square matrix $C(t)$ of degree $s$, such that

$$
P^{(1)}(t)=Q(t) C(t) \quad \text { on } \bar{I}_{1} \cap \bar{I}_{2}
$$

and

$$
\operatorname{rank} C(t)=s \quad \text { on } \bar{I}_{1} \cap \bar{I}_{2},
$$

and every component of $C(t)$ belongs to $K\left(\bar{I}_{1} \cap \bar{I}_{2}\right)$.

Moreover, we have especially

$$
\left(\begin{array}{c}
\hat{\boldsymbol{p}}_{m_{r+1}^{\prime}}(t) \\
\vdots \\
\hat{\boldsymbol{p}}_{m_{n}^{\prime}}(t)
\end{array}\right)=\left(\begin{array}{c}
\hat{\boldsymbol{q}}_{m_{r+1}^{\prime}}(t) \\
\vdots \\
\hat{\boldsymbol{q}}_{m_{n}^{\prime}}(t)
\end{array}\right) C(t) \text { on } \bar{I}_{1} \cap \bar{I}_{2} .
$$

$\S 6$. Proof of Theorem 3 (continued).

If we represent the matrix $C(t)$ in the form of a blocked matrix :

$$
\left.C(t)=\left(\begin{array}{ll}
\overbrace{C_{11}(t)}^{s^{\prime}} & \overbrace{C_{12}(t)}^{s-s^{\prime}} \\
C_{21}(t) & C_{22}(t)
\end{array}\right)\right\} s-s^{\prime},
$$


then, the relation (5.18) and the definition of the vectors $\hat{\boldsymbol{p}}_{m_{\sigma}^{\prime}}(t)$ and $\hat{\boldsymbol{q}}_{m_{\sigma}^{\prime}}(t)$ :

imply

$$
\hat{\boldsymbol{p}}_{m_{\sigma}^{\prime}}(t)=\left(\hat{\boldsymbol{x}}_{m_{\sigma}^{\prime}}(t), \hat{\boldsymbol{y}}_{m_{\sigma}^{\prime}}(t)\right) \text { and } \hat{\boldsymbol{q}}_{m_{\sigma}^{\prime}}(t)=\left(\hat{\boldsymbol{x}}_{m_{\sigma}^{\prime}}(t), \hat{\boldsymbol{z}}_{m_{\sigma}^{\prime}}(t)\right) \text {, }
$$

and

$$
(\sigma=r+1, r+2, \cdots, n)
$$

$$
\left(\begin{array}{c}
\hat{\boldsymbol{x}}_{m_{r+1}^{\prime}}(t) \\
\vdots \\
\hat{\boldsymbol{x}}_{m_{n}^{\prime}}(t)
\end{array}\right)=\left(\begin{array}{c}
\hat{\boldsymbol{x}}_{m_{r+1}^{\prime}}(t) \\
\vdots \\
\hat{\boldsymbol{x}}_{m_{n}^{\prime}}(t)
\end{array}\right) C_{11}(t)+\left(\begin{array}{c}
\hat{\boldsymbol{z}}_{m_{r+1}^{\prime}}(t) \\
\vdots \\
\hat{\boldsymbol{z}}_{m_{n}^{\prime}}(t)
\end{array}\right) C_{21}(t)
$$

$$
\left(\begin{array}{c}
\hat{\boldsymbol{y}}_{m_{r+1}^{\prime}}(t) \\
\vdots \\
\hat{\boldsymbol{y}}_{m_{n}^{\prime}}(t)
\end{array}\right)=\left(\begin{array}{c}
\hat{\boldsymbol{x}}_{m_{r+1}^{\prime}}(t) \\
\vdots \\
\hat{\boldsymbol{x}}_{m_{n}^{\prime}}(t)
\end{array}\right) C_{12}(t)+\left(\begin{array}{c}
\hat{\boldsymbol{z}}_{m_{r+1}^{\prime}}(t) \\
\vdots \\
\hat{\boldsymbol{z}}_{m_{n}^{\prime}}(t)
\end{array}\right) C_{22}(t)
$$

on $\bar{I}_{1} \cap \bar{I}_{2}$.

It is easily seen from the condition (5.13) and the relation (6.1), that

$$
C_{11}(t)=E_{s^{\prime}} \text { and } C_{21}(t)=O \text {, }
$$

where $E_{s^{\prime}}$ is the unit matrix of degree $s^{\prime}$. Hence the matrix $C(t)$ has the following form:

$$
C(t)=\left(\begin{array}{cc}
E_{s} & C_{12}(t) \\
O & C_{22}(t)
\end{array}\right) .
$$

Now let $t_{1}$ be a point belonging to $I_{1} \cap I_{2}$. Then, since $C\left(t_{1}\right)$ is non-singular, there exists a positive number $\hat{\varepsilon}$ such that any square matrix $C$ of degree $s$, satisfying $\left\|C-C\left(t_{1}\right)\right\|<\hat{\varepsilon}$, is non-singular, where $\|\cdot\|$ denotes the Euclidean norm of a matrix.

We can find, in virtue of the continuity of functions, a positive number $\varepsilon_{0}$ such that $\left\|C(t)-C\left(t_{1}\right)\right\|<\hat{\varepsilon}$ whenever $\left|t-t_{1}\right|<\varepsilon_{0}$ and $t \in I_{1} \cap I_{2}$.

Let $t_{1}^{\prime}$ be a point belonging to $I_{1} \cap I_{2}$ such that $0<t_{1}^{\prime}-t_{1}<\varepsilon_{0}$ and let $\varepsilon_{1}$ be a small positive number satisfying the inequality $t_{1}+\varepsilon_{1}<t_{1}^{\prime}-\varepsilon_{1}$.

Furthermore we prepare a real-valued function $\chi(t)$ defined and of class $C^{\infty}$ on $-\infty<t<+\infty$, such that $0 \leqq \chi(t) \leqq 1$ for all $t, \chi(t)=1$ for $t \leqq t_{1}+\varepsilon_{1}$ and $\chi(t)=0$ for $t \geqq t_{1}^{\prime}-\varepsilon_{1}$.

Let $\widetilde{C}(t)$ be a square matrix of degree $s$, defined in the following way:

$$
\tilde{C}(t)= \begin{cases}C(t) & \text { for } \alpha_{2} \leqq t \leqq t_{1}, \\ \chi(t)\left(C(t)-C\left(t_{1}^{\prime}\right)\right)+C\left(t_{1}^{\prime}\right) & \text { for } t_{1} \leqq t \leqq t_{1}^{\prime}, \\ C\left(t_{1}^{\prime}\right) & \text { for } t_{1}^{\prime} \leqq t<+\infty\end{cases}
$$

Since $\left\|\tilde{C}(t)-C\left(t_{1}\right)\right\|<\hat{\varepsilon}$ for $t_{1} \leqq t \leqq t_{1}^{\prime}, \quad \tilde{C}(t)$ is non-singular on $\alpha_{2} \leqq t<+\infty$. Further we can easily verify that every component of $\tilde{C}(t)$ is of class $C^{\mu}$ on $\alpha_{2} \leqq t<+\infty$.

By putting 


$$
\begin{aligned}
& \tilde{C}_{12}(t)=\chi(t)\left(C_{12}(t)-C_{12}\left(t_{1}^{\prime}\right)\right)+C_{12}\left(t_{1}^{\prime}\right), \\
& \tilde{C}_{22}(t)=\chi(t)\left(C_{22}(t)-C_{22}\left(t_{1}^{\prime}\right)\right)+C_{22}\left(t_{1}^{\prime}\right)
\end{aligned}
$$

on $t_{1} \leqq t \leqq t_{1}^{\prime}$, we see that the matrix $\tilde{C}(t)$ has the following form:

$$
\tilde{C}(t)= \begin{cases}\left(\begin{array}{cc}
E_{s^{\prime}} & C_{12}(t) \\
O & C_{22}(t)
\end{array}\right) & \text { for } \alpha_{2} \leqq t \leqq t_{1} ; \\
\left(\begin{array}{cc}
E_{s^{\prime}} & \tilde{C}_{12}(t) \\
O & \tilde{C}_{22}(t)
\end{array}\right) & \text { for } t_{1} \leqq t \leqq t_{1}^{\prime} ; \\
\left(\begin{array}{cc}
E_{s^{\prime}} & C_{12}\left(t_{1}^{\prime}\right) \\
O & C_{22}\left(t_{1}^{\prime}\right)
\end{array}\right) & \text { for } t_{1}^{\prime} \leqq t<+\infty .\end{cases}
$$

If we define a matrix $P^{(2)}(t)$ on $\bar{I}_{1} \cup \bar{I}_{2}$ in the following manner:

$$
P^{(2)}(t)= \begin{cases}P^{(1)}(t) & \text { for } \alpha_{1} \leqq t \leqq \alpha_{2} \\ Q(t) \tilde{C}(t) & \text { for } \alpha_{2} \leqq t \leqq \beta_{2}\end{cases}
$$

then, the matrix $P^{(2)}(t)$ satisfies a linear equation

$$
B(t) P^{(2)}(t)=O \quad \text { on } \bar{I}_{1} \cup \bar{I}_{2},
$$

and satisfies a condition

$$
\operatorname{rank} P^{(2)}(t)=s \quad \text { on } \bar{I}_{1} \cup \bar{I}_{2} \text {, }
$$

and further all components of $P^{(2)}(t)$ belong to $K\left(\bar{I}_{1} \cup \bar{I}_{2}\right)$.

Since it follows from (6.4) and (6.5), that

$$
P^{(2)}(t)= \begin{cases}\left(X(t), X(t) C_{12}(t)+Z(t) C_{22}(t)\right) & \text { for } \alpha_{2} \leqq t \leqq t_{1} \\ \left(X(t), X(t) \tilde{C}_{12}(t)+Z(t) \tilde{C}_{22}(t)\right) & \text { for } t_{1} \leqq t \leqq t_{1}^{\prime} \\ \left(X(t), X(t) C_{12}\left(t_{1}^{\prime}\right)+Z(t) C_{22}\left(t_{1}^{\prime}\right)\right) & \text { for } t_{1}^{\prime} \leqq t \leqq \beta_{2}\end{cases}
$$

if we put

$$
Y(t)\left(\equiv\left(\begin{array}{c}
\hat{\boldsymbol{y}}_{1}(t) \\
\vdots \\
\hat{\boldsymbol{y}}_{n}(t)
\end{array}\right)\right)= \begin{cases}X(t) C_{12}(t)+Z(t) C_{22}(t) & \text { for } \alpha_{2} \leqq t \leqq t_{1} \\
X(t) \widetilde{C}_{12}(t)+Z(t) \widetilde{C}_{22}(t) & \text { for } t_{1} \leqq t \leqq t_{1}^{\prime} \\
X(t) C_{12}\left(t_{1}^{\prime}\right)+Z(t) C_{22}\left(t_{1}^{\prime}\right) & \text { for } t_{1}^{\prime} \leqq t \leqq \beta_{2}\end{cases}
$$

then, by taking the form (5.8) of the matrix $P^{(1)}(t)$ into consideration, we have

$$
P^{(2)}(t)=(X(t), Y(t)) \quad \text { on } \bar{I}_{1} \cup \bar{I}_{2} \text {. }
$$

We shall here show that

$$
\operatorname{rank} Y(t)=s-s^{\prime} \quad \text { on } \bar{I}_{1} \cup \bar{I}_{2} \text {. }
$$

It is already, known that $\operatorname{rank} Y(t)=s-s^{\prime}$ on $\bar{I}_{1}$, and further, as the vectors $\hat{\boldsymbol{y}}_{m}(t)(m=1,2, \cdots, n)$ are of $\left(s-s^{\prime}\right)$-dimension, we see

$$
\operatorname{rank} Y(t) \leqq s-s^{\prime} \quad \text { on } \tilde{I}_{2} \text {. }
$$


ON THE CONSTRUCTION OF LINEARLY INDEPENDENT VECTORS

If there exists a point $t_{0} \in \bar{I}_{2}$ such that $\operatorname{rank} Y\left(t_{0}\right)<s-s^{\prime}$, then we get

$$
\begin{aligned}
\operatorname{rank} P^{(2)}\left(t_{0}\right) & \leqq \operatorname{rank} X\left(t_{0}\right)+\operatorname{rank} Y\left(t_{0}\right) \\
& <s^{\prime}+\left(s-s^{\prime}\right)=s,
\end{aligned}
$$

which contradicts the condition (6.6).

By repeating the above-mentioned process for each pair $\left\{I_{\kappa}, I_{\kappa+1}\right\}(\kappa=2,3, \cdots$, $\left.\kappa_{0}-1\right)$ of intervals, we obtain a matrix:

$$
Y(t)=\left(\boldsymbol{y}_{s^{\prime}+1}(t), \boldsymbol{y}_{s^{\prime}+2}(t), \cdots, \boldsymbol{y}_{s}(t)\right)
$$

defined on the interval $I$, such that

$$
\begin{array}{ll}
\operatorname{rank} Y(t)=s-s^{\prime} & \text { on } I ; \\
\operatorname{rank}(X(t), Y(t))=s & \text { on } I,
\end{array}
$$

and $\boldsymbol{y}_{s^{\prime}+1}(t), \boldsymbol{y}_{s^{\prime}+2}(t), \cdots, \boldsymbol{y}_{s}(t)$ belong to $W(I)$.

The vectors $\boldsymbol{y}_{s^{\prime}+1}(t), \boldsymbol{y}_{s^{\prime}+2}(t), \cdots, \boldsymbol{y}_{s}(t)$ are thus the desired ones.

\section{BIBLIOGRAPHY}

[1] Y. Hirasawa, On solutions of a homogeneous linear matrix equation with variable components, Kodai Math. J., 6 (1983), 70-79.

Department of Mathematics

Tokyo Institute of TECHNOLOGY 